\title{
Organizing Pneumonia Induced by Tocilizumab in a Patient with Rheumatoid Arthritis
}

\author{
Pedro A. Gouveia ${ }^{1}$, Eduarda Carneiro Gomes Ferreira ${ }^{2}$, Paulo M. Cavalcante Neto ${ }^{3}$ \\ 1. Internal Medicine, Hospital Das Clinicas, Federal University of Pernambuco, Recife, BRA 2. Internal Medicine, \\ Hospital Das Clínicas, Federal University of Pernambuco, Recife, BRA 3. Internal Medicine: Pulmonology, Institute of \\ Medical Assistance to the State Public Servant, São Paulo, BRA
}

Corresponding author: Pedro A. Gouveia, pedroalves@doctor.com

\begin{abstract}
Interstitial lung disease is a significant extra-articular manifestation of rheumatoid arthritis, due to its prevalence, morbidity and mortality. Biological therapies are widely used for rheumatoid arthritis treatment. However, some biological agents have been related to the induction or exacerbation of interstitial lung disease. We report a 51-year-old woman with knee arthralgia, hand and foot joint deformities. Although there were no respiratory symptoms, rheumatoid arthritis and interstitial lung disease were diagnosed. High-resolution computed tomography (HRCT) detected a radiological pattern of nonspecific interstitial pneumonia. After tocilizumab therapy for nine months, a second HRCT detected a worsening of interstitial lung disease, presenting a pattern of organizing pneumonia. Tocilizumab was discontinued and prednisone ( $1 \mathrm{mg} / \mathrm{kg} /$ day) was introduced. After two months, a further HRCT detected a significant improvement in organizing pneumonia. There are few similar cases in the literature of tocilizumab-induced organizing pneumonia in patients with rheumatoid arthritis. Despite being a rare adverse effect, knowledge of this association is important for monitoring the use of tocilizumab.
\end{abstract}

Received 12/30/2019 Review began 01/05/2020 Review ended 02/11/2020 Published 02/13/2020

\section{๑) Copyright 2020}

Gouveia et al. This is an open access article distributed under the terms of the Creative Commons Attribution License CC-BY 4.0., which permits unrestricted use, distribution, and reproduction in any medium, provided the original author and source are credited.
Categories: Internal Medicine, Pulmonology, Rheumatology

Keywords: drug-induced pneumonia, rheumatoid arthritis, interstitial lung disease, organizing pneumonia, tocilizumab

\section{Introduction}

Interstitial lung disease (ILD) is a frequent pulmonary manifestation of rheumatoid arthritis (RA) contributing to its morbidity and mortality [1]. ILD may be related to the chronic inflammatory process of RA itself, as well as to the immunomodulation of disease-modifying antirheumatic drugs (DMARDs) used in treatment [2]. Synthetic chemical compounds (csDMARDs) and biological agents (bDMARDs) have been related to the induction or exacerbation of ILD, making it difficult to choose a safe and effective therapeutic approach $[2,3]$. We report a RA patient who developed organizing pneumonia (OP) after treatment with tocilizumab (TCZ), an interleukin-6 (IL-6) receptor blocking monoclonal antibody.

\section{Case Presentation}

A 51-year-old woman referred to an outpatient clinic due to bilateral knee arthralgia, severe joint pain in the proximal metacarpophalangeal and interphalangeal joints with 30-minute morning stiffness. Although she had smoked until seven years ago, she denied respiratory symptoms. Her physical examination revealed wrist and metacarpophalangeal joint blocking, ulnar drift of the fingers, swan neck deformities, and fibular drift of the toes. Rales were audible in lower thirds of the lung fields, and the oxygen saturation was $97 \%$. Laboratory tests revealed rheumatoid factor $110 \mathrm{U} / \mathrm{mL}$ (normal, $<20 \mathrm{U} / \mathrm{mL}$ ), anticitrullinated protein antibody $57 \mathrm{U}$ (normal, <10 U), C-reactive protein $13 \mathrm{mg} / \mathrm{dL}$ (normal, < $0.5 \mathrm{mg} / \mathrm{dL}$ ) and the erythrocyte sedimentation rate $97 \mathrm{~mm}$ /hour. RA diagnosis was performed with the Disease Activity Score (DAS28) 4.03, corresponding to a moderate disease activity.

High-resolution computed tomography (HRCT) was suggestive of nonspecific interstitial pneumonia (NSIP) (Figure $1 A-C$ ). Spirometry revealed a restrictive disorder with a forced vital capacity (FVC) of $46 \%$ of the predicted level, a forced expiratory volume in the first second (FEV1) of $48 \%$ of the predicted value and a FEV1/FVC ratio above the lower limit of normality. Bronchoscopy with bronchoalveolar lavage culture for bacteria and fungi and polymerase chain reaction for Mycobacterium tuberculosis were negative. RA-ILD diagnosis was made after excluding other causes and treatment was initiated due to the severity of the findings. We opted for a monthly dose of TCZ ( $8 \mathrm{mg} / \mathrm{kg}$ ) and prednisone $0.5 \mathrm{mg} / \mathrm{kg} / \mathrm{day}$, as several csDMARDs and bDMARDs were associated with the induction or exacerbation of lung disease. The patient presented an improvement of joint symptoms and low disease activity with DAS28 of 2.8, with a gradual withdrawal of prednisone. 


\section{Cureus}

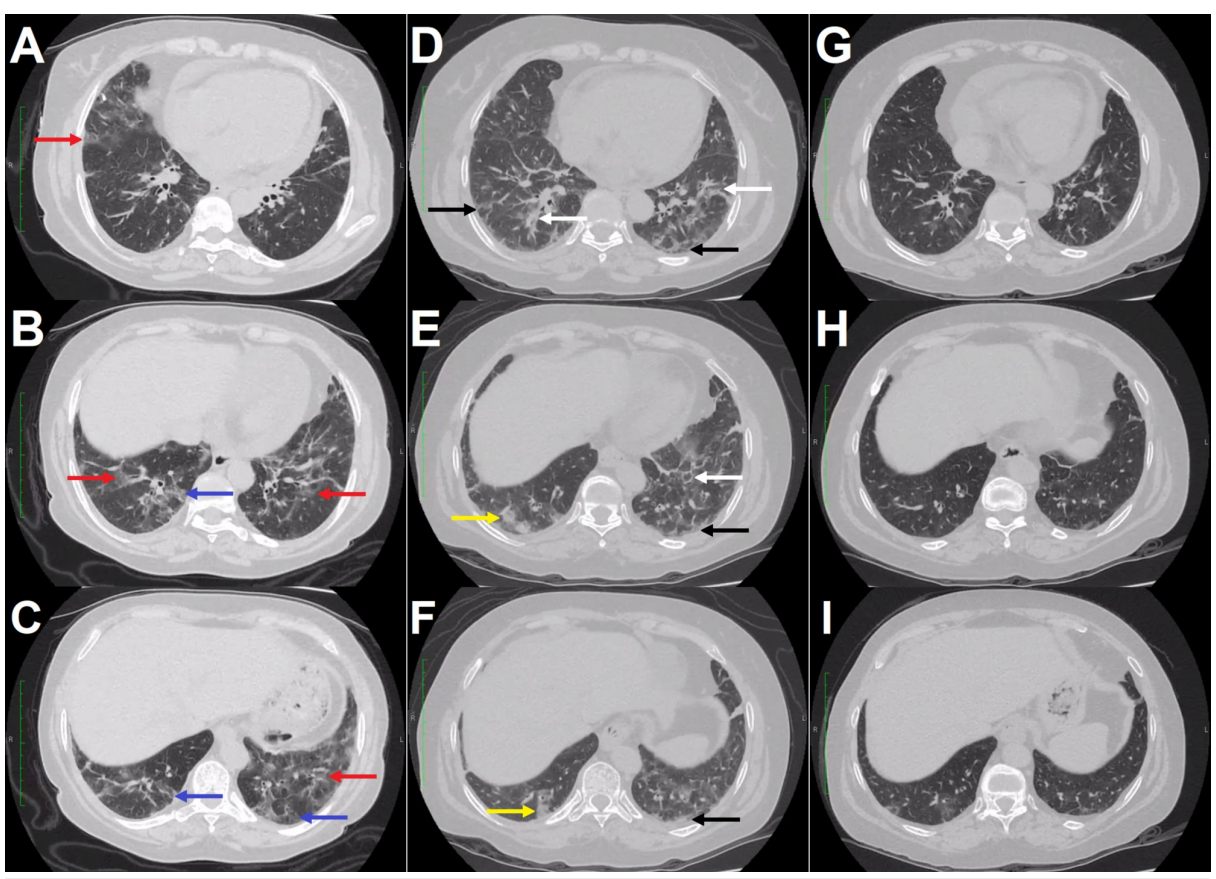

FIGURE 1: High-resolution computed tomography images of the patient with rheumatoid arthritis.

Ground-glass opacities (red arrows) predominant in the lower third of the lungs, associated with mild traction bronciolectasis (blue arrows), suggesting nonspecific interstitial pneumonia (A-C). Increased areas of ground-glass opacities of a predominantly perilobular and peribronchovascular pattern (white arrows), associated with reticulation (black arrows) and bilateral patchy consolidations (yellow arrows), more evident in lower lobes, suggestive of organizing pneumonia (D-F). Significant reduction in ground-glass and consolidative opacities after tocilizumab suspension and cortiscorteroids onset (G-I).

After nine months of continuous TCZ therapy, a chest HRCT was performed and was suggestive of OP (Figure 1D-F). A bronchoscopy with bronchoalveolar lavage culture detected no infection. New spirometry also demonstrated restrictive disorder with lower values of both FVC and FEV1 (44\% and 37\% predicted, respectively). In view of the significant worsening of the radiological pattern for OP, TCZ therapy was discontinued. Prednisone $1 \mathrm{mg} / \mathrm{kg} / \mathrm{day}$ was introduced to OP treatment. After two months, a new chest HRCT was performed and presented a significant improvement in findings compared to the previous examination (Figure 1G-I). Prednisone was gradually reduced and tofacitinib initiated due to joint activity. The patient had a good response to treatment with joint and pulmonary improvement.

\section{Discussion}

Pulmonary manifestations of RA usually occur in the first five years of disease and in some cases precede joint symptoms $[4,5]$. Patients with RA may have low functional capacity due to joint deformities, and therefore do not develop respiratory symptoms, such as the current case. Crackles in the lower third of the lungs on physical examination led us to investigate ILD. Initial diagnosis of RA-ILD is important, since methotrexate (MTX), a first-line drug in RA treatment, may increase a small risk for interstitial pneumonitis $[2,3]$. Therefore, physicians should be aware of diagnosing ILD in all patients with RA, and not be restricted to those who are symptomatic.

RA-ILD presents several histopathological and radiological patterns. The most frequent patterns are usual interstitial pneumonia in $40 \%-62 \%$ and NSIP in $11 \%-38 \%[3,4]$. OP is a less common pattern and may reach $11 \%$ of RA-ILD [4]. There is no clear difference in clinical features or radiological findings between OP secondary to RA and cryptogenic organizing pneumonia (COP). Initial symptoms are usually dyspnea, cough and constitutional symptoms. Most common HRCT findings are bilateral involvement with random peripheral and central lesions in the middle and lower third of the lungs. The following radiological patterns are most identified in OP: ground-glass opacities (88.9\%), consolidation (83.3\%) and peribronchovascular opacities (52.8\%) [6]. Lung biopsy is the gold standard for COP diagnosis. However, association of clinical and radiological findings typical of OP in RA patients is sufficient for diagnosis $[5,7]$.

Pérez-Dórame et al. reported a correlation between RA activity scores and the ground-glass score present on chest HRCTs, suggesting that inflammation in RA-ILD is secondary to RA disease activity [8]. Akiyama et al. corroborated this hypothesis proving the relationship between the acute exacerbation of RA-ILD and uncontrolled arthritis activity [9]. In the present case, the patient improved from joint inflammation and 
presented low DAS28 activity after TCZ initiation. This response to treatment suggests ILD exacerbation was due to bDMARD rather than RA activity.

New-onset and exacerbation of ILD have been reported in association with several biological therapies [1]. Tumor necrosis factor (TNF) inhibitors stand out, affecting up to 97\% of bDMARD-associated ILD cases. A relation between TCZ, an IL-6 receptor blocking monoclonal antibody, and ILD is not yet universally established in the literature. However, a systematic review conducted in 2011 identified non-infectious pulmonary adverse effects in six cases (1.0\%) amongst 589 patients undergoing treatment with TCZ for RA [10].

Several case reports suggest that OP may be caused by toxicity of bDMARDs [11-13]. Mori et al. identified 21 Japanese patients with OP and RA. Twelve patients developed OP after biological onset, one with TCZ and the others with TNF inhibitors [7]. A causal relationship between drug toxicity and lung disease is reinforced because most patients had control of disease activity. Similarly, the patient reported here presented low activity in DAS28.

Two cases of TCZ-associated OP have been previously reported in RA patients. In the first case, Ikegawa et al. described a 66-year-old woman with RA who initiated TCZ after MTX therapy for two years. Three days after the first TCZ infusion, she was diagnosed with OP and elevated transaminases, which improved after discontinuing TCZ and initiating corticosteroid [12]. In the second case, Mori et al. identified a 63-year-old man with RA activity controlled by TCZ therapy for 25 months, in combination with MTX and tacrolimus. OP was diagnosed and TCZ was withdrawn with prednisolone $30 \mathrm{mg}$ /day inducing a recovery from ILD [7]. The current case is the third to describe TCZ-associated OP in RA patients; however, we present an exacerbation of a previous ILD. Use of the Naranjo adverse drug reaction probability scale indicates the strength of the causal relationship as probable in the present case [14].

All three TCZ-associated OP cases presented excellent responses by discontinuing TCZ and introducing corticosteroid therapy [7,12]. OP is known to respond better to corticosteroids than other RA-ILD subtypes [1]. RA reactivation is a concern after TCZ has been discontinued. Reintroducing biological therapy is difficult, and it is therefore more prudent to consider using another bDMARD. After the 12 cases of improved OP associated with bDMARD, Mori et al. maintained three patients with low disease activity without medication and introduced another bDMARD or MTX in the remaining cases [7]. Although MTX may increase a small risk for interstitial pneumonitis, Kiely et al. recently identified no association between MTX exposure and RA-ILD incidence in two cohorts (ERAS and ERAN) [2,3,15]. We chose to introduce tofacitinib because there is no association described with ILD.

\section{Conclusions}

We report a TCZ-associated OP case in a patient with RA, with few similar cases described in the literature. Even with pulmonary severity, interruption of TCZ and corticosteroid initiation were enough to resolve this case. For better elucidation of TCZ lung safety, clinical trials comparing efficacy and safety of different bDMARDs in this clinical setting are required. Despite being a rare adverse effect, knowledge of this association is important for monitoring the use of TCZ.

\section{Additional Information \\ Disclosures}

Human subjects: Consent was obtained by all participants in this study. Comitê de Ética do Hospital das Clínicas da Universidade Federal de Pernambuco - HC/UFPE issued approval 10189519.3.0000.8807. Conflicts of interest: In compliance with the ICMJE uniform disclosure form, all authors declare the following: Payment/services info: All authors have declared that no financial support was received from any organization for the submitted work. Financial relationships: All authors have declared that they have no financial relationships at present or within the previous three years with any organizations that might have an interest in the submitted work. Other relationships: All authors have declared that there are no other relationships or activities that could appear to have influenced the submitted work.

\section{References}

1. Bes C: Comprehensive review of current diagnostic and treatment approaches to interstitial lung disease associated with rheumatoid arthritis. Eur J Rheumatol. 2019, 6:146-149.

2. Diamanti AP, Markovic M, Argento G, et al.: Therapeutic management of patients with rheumatoid arthritis and associated interstitial lung disease: case report and literature review. Ther Adv Respir Dis. 2017, 11:6472. 10.1177/1753465816668780

3. Hallowell RW, Horton MR: Interstitial lung disease in patients with rheumatoid arthritis: spontaneous and drug induced. Drugs. 2014, 74:443-450. 10.1007/s40265-014-0190-z

4. Lee H, Kim DS, Yoo B, et al.: Histopathologic pattern and clinical features of rheumatoid arthritis-associated interstitial lung disease. Chest. 2005, 127:2019-2027. 10.1378/chest.127.6.2019

5. Shaw M, Collins BF, Ho LA, Raghu G: Rheumatoid arthritis-associated lung disease . Eur Respir Rev. 2015, 24:1-16. 10.1183/09059180.00008014 
6. Faria IM, Zanetti G, Barreto MM, et al.: Organizing pneumonia: chest HRCT findings. J Bras Pneumol. 2015, 41:231-237. 10.1590/S1806-37132015000004544

7. Mori S, Koga Y, Sugimoto M: Organizing pneumonia in rheumatoid arthritis patients: a case-based review . Clin Med Insights Circ Respir Pulm Med. 2015, 9:69-80. 10.4137/CCRPM.S23327

8. Pérez-Dórame R, Mejía M, Mateos-Toledo H, Rojas-Serrano J: Rheumatoid arthritis-associated interstitial lung disease: lung inflammation evaluated with high resolution computed tomography scan is correlated to rheumatoid arthritis disease activity. Reumatol Clin. 2015, 11:12-16. 10.1016/j.reuma.2014.02.007

9. Akiyama M, Kaneko Y, Yamaoka K, Kondo H, Takeuchi T: Association of disease activity with acute exacerbation of interstitial lung disease during tocilizumab treatment in patients with rheumatoid arthritis: a retrospective, case-control study. Rheumatol Int. 2016, 36:881-889. 10.1007/s00296-016-3478-3

10. Hadjinicolaou AV, Nisar MK, Bhagat S, Parfrey H, Chilvers ER, Östör AJK : Non-infectious pulmonary complications of newer biological agents for rheumatic diseases: a systematic literature review. Rheumatology. 2011, 50:2297-2305. 10.1093/rheumatology/ker289

11. Soubrier M, Jeannin G, Kemeny JL, et al.: Organizing pneumonia after rituximab therapy: two cases . Joint Bone Spine. 2008, 75:362-365. 10.1016/j.jbspin.2007.10.009

12. Ikegawa K, Hanaoka M, Ushiki A, Yamamoto H, Kubo K: A case of organizing pneumonia induced by tocilizumab. Intern Med. 2011, 50:2191-2193. 10.2169/internalmedicine.50.5497

13. Cho SK, Oh IH, Park CK, Bae SC, Sung YK: Etanercept induced organizing pneumonia in a patient with rheumatoid arthritis. Rheumatol Int. 2012, 32:1055-1057. 10.1007/s00296-009-1350-4

14. Naranjo CA, Busto U, Sellers EM, et al.: A method for estimating the probability of adverse drug reactions . Clin Pharmacol Ther. 1981, 30:239-245. 10.1038/clpt.1981.154

15. Kiely P, Busby AD, Nikiphorou E, et al.: Is incident rheumatoid arthritis interstitial lung disease associated with methotrexate treatment? Results from a multivariate analysis in the ERAS and ERAN inception cohorts. BMJ Open. 2019, 9:e028466. 10.1136/bmjopen-2018-028466 